\title{
Prevalence of Toxoplasma gondii in pet and stray cats in Klang Valley, Malaysia
}

\author{
Tan, L.P., Megat Abd. Rani, P.A. ${ }^{1}$, Sharma, R.S.K. ${ }^{2}$, Syed Hussain, S.S. ${ }^{3}$ and Watanabe, M. ${ }^{*}$ \\ ${ }^{1}$ Department of Companion Animal Medicine and Surgery, Faculty of Veterinary Medicine, Universiti Putra \\ Malaysia, 43400 UPM Serdang, Selangor, Malaysia \\ ${ }^{2}$ Department of Veterinary Laboratory Diagnostics, Faculty of Veterinary Medicine, Universiti Putra Malaysia, \\ 43400 UPM Serdang, Selangor, Malaysia \\ ${ }^{3}$ Department of Veterinary Clinical Studies, Faculty of Veterinary Medicine, Universiti Putra Malaysia, 43400 \\ UPM Serdang, Selangor, Malaysia \\ *Corresponding author e-mail: maraika@upm.edu.my \\ Received 7 August 2019; received in revised form 29 June 2020; accepted 1 July 2020
}

\begin{abstract}
Toxoplasma gondii, a zoonotic protozoan that has a worldwide distribution, is known to infect many warm-blooded vertebrates. The feline species including domestic cats are the definitive hosts for Toxoplama gondii and shed the infective oocyst. There is lack of information on the prevalence of Toxoplasma gondii in cats in Malaysia. The objective of this study was to determine both the seroprevalence of $T$. gondii and the prevalence of $T$. gondii DNA in cats' feces in Klang Valley, Malaysia. 198 blood and 201 fecal samples were collected from pet and stray cats from the local council, Dewan Bandaraya Kuala Lumpur (DBKL) and University Veterinary Hospital, Universiti Putra Malaysia respectively. The overall seroprevalence of Toxoplasma gondii in cats in the Klang Valley was found to be $5.5 \%$. There was a high prevalence (10.5\%) of T. gondii DNA detected in the cat fecal samples in both pet and stray cats suggestive of $T$. gondii oocyst shedding. Stray cats showed a higher seroprevalence and molecular prevalence of $T$. gondii than the pet cats. However, comparative analysis using $C h i$-square test showed no significant difference between both groups $(\mathrm{P}>0.05)$. Higher prevalence (10.5\%) of cats shedding T. gondii DNA as compared to the seroprevalence (5.5\%) was found in the cat population in the Klang Valley. The high prevalence of cats shedding T. gondii DNA is alarming as this may directly reflect the number of oocysts excreted into the environment posing a significant public health hazard.
\end{abstract}

\section{INTRODUCTION}

Toxoplasma gondii is the only species that belongs in the genus Toxoplasma. T. gondii is a protozoan parasite that infects most mammalian species including cats, dogs, cattle as well as humans. It is a major human health concern in many countries as it causes clinically important diseases (RobertGangneux and Dardé, 2012). Seroprevalence of toxoplasmosis in humans has been reported between 3\%-70\% in Southeast Asian (SEA) countries (Nissapatorn, 2007). In Malaysia, prevalence of T. gondii in healthy humans can be up to $30.2 \%$ and $33 \%$ in children (Nissapatorn, 2007). Pregnant women as well as immunocompromised patients have been reported to have higher seroprevalence rates of $T$. gondii of up to 49\% (Nissapatorn et al., 2003; Nissapatorn, 2007; Andiappan et al., 2014).

The main cause of toxoplasmosis in humans was found to be due to consumption of raw meat as well as frequent contact with cats (Nissapatorn, 2007; Andiappan et al., 2014; Brandon-Mong et al., 2015). A study in Klang Valley, Malaysia in 2015 showed that pet owners and veterinary technicians had a seroprevalence between 30-33\% (BrandonMong et al., 2015). Frequent contact with cats increases the risk of zoonotic transmission as domestic cats will shed T. gondii oocyst 
up to 21 days upon initial infection (Lilly and Wortham, 2013; Lappin et al., 2015). Reshedding of $T$. gondii in cats has also been reported (Dubey et al., 2009; Elmore $e t$ al., 2010).

Toxoplasma gondii completes its life cycle in the domestic cats (Cerro et al., 2014). It rarely causes clinical manifestations however, clinical cases are seen in immunocompromised cats (Dubey et al., 2009; Dabritz and Conrad, 2010). Clinical manifestations frequently observed in cats include ocular toxoplasmosis and congenital toxoplasmosis, some with severe respiratory and neurological signs (Dubey et al., 2009). Severe clinical cases are usually seen in kittens infected transplacentally (Dubey $e t$ al., 2009).

Due to the short oocyst shedding period, the prevalence of oocysts found in fecal samples tends to be low. Approximately, only about $1 \%$ of cats in a population are found to be shedding oocysts at any given time (Hill and Dubey, 2002; Elmore et al., 2010). A high prevalence of $6 \%$ of cats shedding T. gondii oocyst was found in a study in the United States (Lilly and Wortham, 2013). Various laboratory methods have been used to diagnose toxoplasmosis such as microscopic detection, bioassay in mice and PCR (Hooshyar et al., 2007; Salant et al., 2007; Lilly and Wortham, 2013; Jung et al., 2015). Microscopic detection of oocyst is less reliable than bioassay and PCR as $T$. gondii oocyst is morphologically similar to Hammondia hammondi and Besnoita spp. (Dubey et al., 2009; Györke et al., 2011). On the other hand, bioassay in mice is a more time consuming diagnostic method as compared to PCR (Salant et al., 2010; Saki and Khademvatan, 2014). Polymerase chain reaction is deemed to be the most sensitive, specific and convenient test for $T$. gondii oocyst detection (Salant et al., 2010; Zewdu et al., 2015). Serological tests of T. gondii are more commonly used as the diagnostic method for infection (Dubey et al., 2009). Studies across the world estimated seroprevalence of $T$. gondii in domestic cats with a wide range, between 11-91\% (Jittapalapong et al., 2007; Lee et al., 2011; Castillo-Morales et al., 2012; Opsteegh et al., 2012; Garcia et $a l ., 2014$; Liu et al., 2014). The huge range of seroprevalence of Toxoplasma gondii may be due to different serological assays used in each individual study (Vollaire $e t$ al., 2005; Millán et al., 2009; Castillo-Morales et al., 2012; Oi et al., 2015). Enzyme-linked immunosorbent assays were shown to have a higher sensitivity and specificity in comparison with other serological assays such as indirect immunofluorescent antibody test (IFAT) and modified agglutination test (MAT) in recent studies (Györke et al., 2011; Paştiu et al., 2015). There is scant data regarding the seroprevalence of $T$. gondii in cats in Malaysia. Only one study has been conducted in the pet cat population in Ipoh, Malaysia using IFAT which detected a seroprevalence of $14.5 \%$ (Chandrawathani et al., 2008). Furthermore, there is no study on the prevalence of $T$. gondii oocyst in the cat fecal samples using molecular detection techniques. Thus, the objectives of the present study were to determine the presence of T. gondii in pet and stray cats in the Klang Valley, Malaysia and to determine the risk factors that may be involved.

\section{METHOD}

\section{Study design}

This cross-sectional study involved 101 pet cats and 100 stray cats. Cats that were owned and kept indoors or allowed to roam outdoors were categorized as pet cats, whereas cats without owners were considered as stray cats. Pet cat samples included those presented to University Veterinary Hospital, Universiti Putra Malaysia. Stray cat samples were obtained from the local council (Dewan Bandaraya Kuala Lumpur). The age, sex and management of both the pet and stray cats were noted upon collection. Management was only noted for the pet cat population if they were kept totally indoors or allowed to roam outdoors as well as their household management of either kept in a single or multicat household. This study was approved by the Institutional Animal Care and Use Committee, Universiti Putra Malaysia (AUPR088/2016). 


\section{Sample collection}

Blood from 198 cats were collected via jugular venipuncture in plain tubes and were centrifuged at 5000rpm for 5 minutes to separate the serum. Serum samples were kept in $-20^{\circ} \mathrm{C}$ until further use. Fecal samples were collected from 101 pet cats and 100 stray cats using a fecal swab. Fecal samples collected were then placed in 100ul lysis buffer and kept at $-20^{\circ} \mathrm{C}$ for storage until use.

\section{Serological analysis}

A total of 198 (100 pet cats and 98 stray cats) serum samples collected were screened for IgG antibodies against Toxoplasma gondii using indirect-ELISA ID Screen ${ }^{\circledR}$ Toxoplasmosis Indirect Multispecies (ID.vet Innovative Diagnostics, Grabels, France). The optical density of each sample was read under 450nm using a microplate reader TECAN Infinite ${ }^{\circledR}$ 200M Pro (Tecan Group Ltd, Mannedorf, Switzerland). The sample/positive ratio (S/P \%) were calculated using the following formula

$$
\mathrm{S} / \mathrm{P} \%=\frac{O D_{\text {sample }}-O D_{N C}}{O D_{P C}-O D_{N C}} \times 100
$$

According to the manufacturer's guidelines $\mathrm{S} / \mathrm{P}$ values that were greater than $50 \%$ were considered positive.

\section{Molecular analysis}

DNA was extracted from the fecal samples using QIAamp stool mini kit (QIAGEN, Hilden, Germany) following the manufacturer's instructions. Extracted DNA was subjected to nested-PCR to amplify the $T$. gondii B1 gene with two sets of primers that produces a product size of 90bp (Jones et al., 2000). First amplification reaction mixture consisted of $5 \mu \mathrm{L}$ of Promega $5 \mathrm{x}$ Green GoTaq ${ }^{\circledR F l e x i ~ B u f f e r ~(P r o m e g a ~ C o r p o r a t i o n, ~}$ Madison,USA). $1 \mathrm{mM} \mathrm{MgCl}_{2}, 0.1 \mathrm{mM}$ dNTP mix, $1.25 \mathrm{U}$ of Taq polymerase with $0.1 \mathrm{pmoL}$ of both first set of primers and $5 \mu \mathrm{L}$ of sample DNA with a total volume of $25 \mu \mathrm{L}$. $1 \mu \mathrm{L}$ of the first PCR product was then used in the second PCR amplification mix which included $5 \mu \mathrm{L}$ of Promega 5x Green GoTaq ${ }^{\circledR F l e x i ~ B u f f e r, ~}$ $1.5 \mathrm{mM}, 0.1 \mathrm{mM}$ of dNTP mix, $1 \mathrm{U}$ of Taq polymerase and $0.5 \mathrm{pmoL}$ of second set of primers with a total volume of $25 \mu \mathrm{L}$. Both amplification conditions were as used by Jones et al., 2000. Products were then loaded on $2 \%$ agarose gel for gel electrophoresis. Gel was stained with ethidium bromide and viewed under Biorad Gel Doc ${ }^{\mathrm{TM}} \mathrm{XR}$ (Biorad, Hercules, CA, United States). Positive samples were then purified using Qiagen PCR purification kit (QIAGEN, Hilden, Germany) and were sent for sequencing.

\section{Statistical analysis}

Samples were grouped into different risk factors which included age, sex, and management of the cats. Comparative analysis of each group was performed using $C h i$-square test and Fisher exact test. Statistical analysis was performed using IBM SPSS Statistics 22 (IBM, Greenville, United States). The differences were considered statistically significant when $\mathrm{P}<0.05$.

\section{RESULTS}

\section{Seroprevalence of $T$. gondii in pet and stray cats}

The overall seroprevalence in the cat population was 5.6\% (11/198). Among serum samples from the stray cats, 7 out of 98 were positive for IgG antibodies with a seroprevalence of $7.1 \%$. In the pet cat population, 4 samples were positive with a seroprevalence of $4 \%$, calculated at a $95 \%$ confidence interval. Stray cats showed a higher seroprevalence as compared to the pet cats however, Chi-square test demonstrated no significant difference between the two groups. All 4 positive samples in the pet cat population were from cats 1 year old and above, and there were no positives among the kitten and junior cats less than 1 year old. Despite the difference, Fisher exact test showed no significant difference between these two groups $(\mathrm{OR}=0.177 ; 95 \%=0.020,1.570 ; \mathrm{P}>$ 0.05). Age of the stray cats also showed no 
significant association with seropositivity $(\mathrm{OR}=2.200 ; 95 \%=0.388,12.483 ; \mathrm{P}>0.05)$. Comparative analysis using Fisher exact test showed no significant difference between the sex of the cats in both the pet and stray cat population $(\mathrm{P}>0.05)$. Management of the pet cat population also showed no significant association with the seropositivity of toxoplasmosis $(\mathrm{P}>0.05)$. Results are listed as in Table 1 and Table 2.
Molecular prevalence of Toxoplasma gondii in feces of pet and stray cats

Fecal samples of 201 cats (100 stray cats and 101 pet cats) were collected to detect Toxoplasma gondii DNA. A total prevalence of 10.5\% (21/200) for both pet and stray cats positive for T. gondii was obtained. A high prevalence of $15 \%(15 / 100)$ was found in stray cats and $6 \%(6 / 100)$ in pet cats. Positive samples are as shown in Figure 1. All samples

Table 1. P value, odd ratio, confidence interval for sex, age, management of the pet cat sampled for seroprevalence $T$. gondii

\begin{tabular}{lcccc}
\hline & P value & Odd ratio & \multicolumn{2}{c}{ Confidence interval } \\
\cline { 4 - 5 } & & & Lower & Upper \\
\hline Sex & 0.097 & 0.162 & 0.018 & 1.442 \\
Age & 0.135 & 0.177 & 0.018 & 1.774 \\
Indoor/Outdoor & 0.282 & 2.667 & 0.286 & 24.896 \\
$\begin{array}{l}\text { Single/Multi } \\
\text { cat household }\end{array}$ & 0.363 & 3.318 & 0.277 & 39.732 \\
\hline
\end{tabular}

Table 2. $\mathrm{P}$ value, odd ratio, confidence interval for sex and age of the stray cat sampled for seroprevalence T. gondii

\begin{tabular}{lcccc}
\hline & & & \multicolumn{2}{c}{ Confidence interval } \\
\cline { 4 - 5 } & P value & Odd ratio & Lower & Upper \\
\hline Sex & 0.439 & 2.446 & 0.451 & 13.261 \\
Age & 1.000 & 1.289 & 0.271 & 6.129 \\
\hline
\end{tabular}

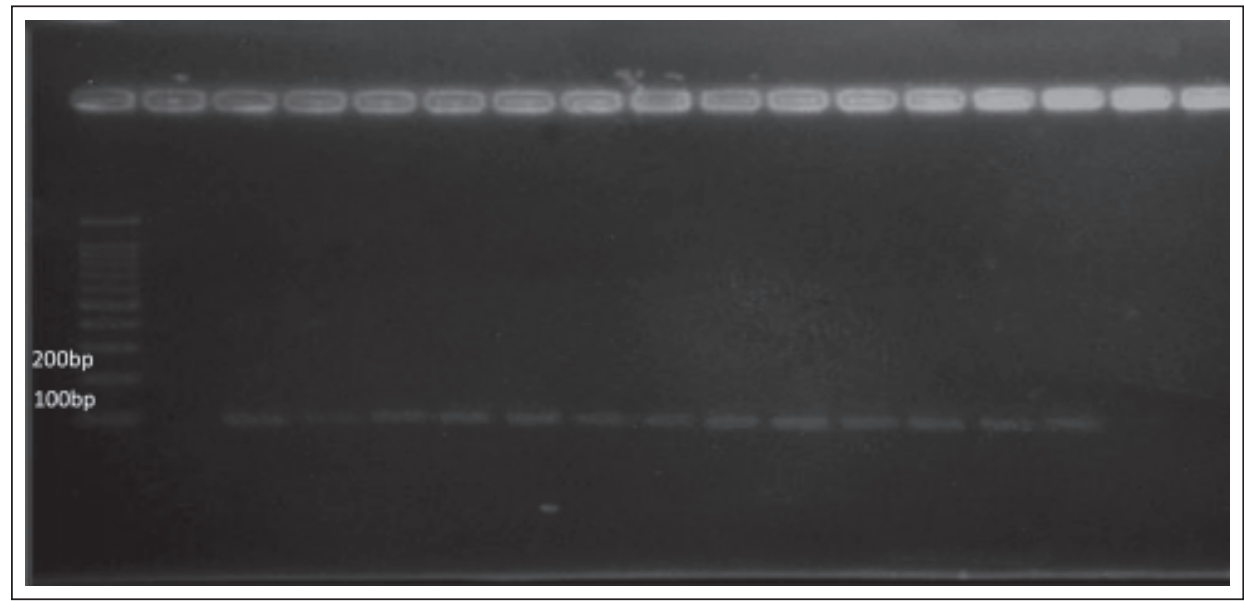

Figure 1. PCR amplification of B1 gene from the cat fecal samples. This figure illustrates positive samples at 96bp size. 
Table 3. P value, odd ratio, confidence interval for sex, age, management of the pet cat sampled for shedding T. gondii oocyst

\begin{tabular}{lcccc}
\hline & P value & Odd ratio & \multicolumn{2}{c}{ Confidence interval } \\
\cline { 3 - 5 } & & & Lower & Upper \\
\hline Sex & 1.000 & 0.958 & 0.184 & 4.993 \\
Age & 1.000 & 0.811 & 0.129 & 5.103 \\
Indoor/Outdoor & 0.207 & 0.300 & 0.052 & 1.929 \\
$\begin{array}{l}\text { Single/Multi } \\
\text { cat household }\end{array}$ & 1.000 & 1.167 & 0.133 & 10.221 \\
\hline
\end{tabular}

Table 4. $\mathrm{P}$ value, odd ratio, confidence interval for sex and age of the stray cat sampled for shedding T. gondii oocyst

\begin{tabular}{lcccc}
\hline & & & \multicolumn{2}{c}{ Confidence interval } \\
\cline { 4 - 5 } & & Odd ratio & Lower & Upper \\
\hline Sex & 0.585 & 1.348 & 0.460 & 3.956 \\
Age & 0.280 & 0.513 & 0.151 & 1.749 \\
\hline
\end{tabular}

sequenced were compared with those in the GenBank database using BLAST (Basic Local Alignment Search Tool). All positive samples were matched and confirmed to be Toxoplasma gondii. Toxoplasma gondii isolates that were sequenced showed 98$100 \%$ similarity to the isolates from small ruminants in Mexico, KX2770388 and Bangladesh KU900748. Statistical analysis using $C h i$-square test showed no significant difference between sex, age of the cats as well as management of the cats for both pet and stray cats. The results are as in Table 3 and Table 4.

\section{DISCUSSION}

The overall seroprevalence for Toxoplasma gondii in cats in the Klang Valley was found to be $5.6 \%$. This seroprevalence is comparatively lower compared to several other studies using Indirect-ELISA (Györke et al., 2011; Castillo-Morales et al., 2012; Opsteegh et al., 2012; Deksne et al., 2013; Rengifo-herrera et al., 2017). Previously a study in Ipoh, Perak Malaysia showed a higher seroprevalence of $14.5 \%$ (Chandrawathani et al., 2008) and in neighboring
Thailand 11.7\% (Jittapalapong et al., 2007) and Philippines 22\% (Garcia et al., 2014). The difference in seroprevalence in different countries has been suggested due to different climate and geographical location of each study conducted as well as serological tools used in the different studies. However the ELISA used in the current study has high sensitivity (96.8\%) and specificity (96.1\%) for the detection of antibodies against $T$. gondii in the feline species (Györke et al., 2011) therefore the low seroprevalence obtained in this study may be an indication of either actual decrease in number of infected cats in the region or recent infections where the cats have yet to seroconvert.

This study demonstrated a high prevalence of $10.5 \%$ T. gondii detected in the cat fecal samples in the Klang Valley. Detection of $T$. gondii in cat feces using PCR is usually assumed to be difficult due to the short oocyst shedding period, and a few studies have demonstrated lower prevalence rates between 0-6\% (Mancianti et al., 2010; Lilly and Wortham, 2013; Jung et al., 2015). However, there is one study that showed a high prevalence of $19.4 \%$ in cat fecal samples in Ethiopia (Dubey et al., 2013). Therefore again the prevalence rates 
appear to differ based on geographical location and the degree of exposure of cats to the protozoa.

Stray cats in the Klang Valley demonstrated a molecular prevalence of $15 \%$ which was much higher than that in pet cats (6\%). Similarly, seroprevalence of $T$. gondii was higher in stray cats compared to the pet cats in the Klang Valley. This finding is similar to other studies where feral or stray cats generally showed a higher seroprevalence than pet cats (Gauss et al., 2003; Nutter et al., 2004; Vollaire et al., 2005; Hooshyar et $a l ., 2007$; Liu et al., 2014). Higher seroprevalence in stray cats may be due to constant exposure to the outdoor environment with higher chances of coming in contact with soil contaminated with $T$. gondii oocysts. Stray cats also have higher risk of ingesting tissue cysts from infected rodents, small mammals as well as wild birds (Gauss et al., 2003; Jung et al., 2015). Thus, pet cats that are allowed to roam outdoors have higher chances of getting $T$. gondii, as seen in this study where 4 out of 6 roaming cats positive for T. gondii in their feces. However, statistical analysis showed no significant difference $(\mathrm{P}>0.05)$ between stray and pet cats. This could be due to the sampled pet population in this study as $60 \%$ of the sampled cats were managed indoor.

The present study showed a low seroprevalence of IgG antibodies against $T$. gondii in the cat population of Klang Valley but in contrast, a high molecular prevalence of $T$. gondii detected from the feces of the cats. Seropositivity of T. gondii has been shown to increase with the age of cats in several studies (Must. K et al., 2017; Wang et $a l ., 2017)$. The low seroprevalence in the present study may be due to the age of the sampled cats. $84 \%$ of the samples were collected from cats aged 6 years and below. Cats aged above 10 years old was found to be a significant risk factor for $T$. gondii seropositivity (Györke et al., 2011). Eventhough, comparative analysis using $\mathrm{Chi}$ square test showed no significant difference $(\mathrm{P}>0.05)$ in this study, older cats showed higher seropositivity as all seropositive samples were cats 1 year old and above. IgG antibodies against $T$. gondii in cats has been reported to appear around 1 to 3 weeks after the initial infection usually regarded as chronic infections. (Robert-Gangneux and Dardé, 2012). All cats that were shedding T. gondii DNA in the fecal samples were negative for IgG antibodies when tested in this study, which suggests that those cats that were PCR positive had not yet seroconverted. Thus, this could suggest that these cats have been recently infected and might be shedding T. gondii oocyst at that point of time (RobertGangneux and Dardé, 2012; Lilly and Wortham, 2013; Lappin, 2015).

In conclusion, the present study demonstrated a high $T$. gondii molecular prevalence of $10.5 \%$ among cats in the Klang Valley, Malaysia. $5.5 \%$ of the cat population in the Klang Valley were seropositive for IgG antibodies against $T$. gondii. The high prevalence of cats possibly shedding $T$. gondii DNA is alarming as this may indicate the number of oocysts directly excreted into the environment increasing the risk of zoonotic disease transmission.

Acknowledgement. This study was funded in part by UPM-IPS grant (9528500) sincere appreciation to Dr. Laryssa Howe from The Institute of Veterinary, Animal and Biomedical Sciences (IVABS), Massey University, Palmerston North for providing the positive control of Toxoplasma gondii for this study.

\section{REFERENCE}

Ahmad, N., Ahmed, H., Irum, S. \& Qayyum, M. (2014). Seroprevalence of IgG and IgM antibodies and associated risk factors for toxoplasmosis in cats and dogs from sub- tropical arid parts of Pakistan 31(4): 777-784.

Andiappan, H., Nissapatorn, V., Sawangjaroen, N., Nyunt, M.H., Lau, Y., Khaing, S.L., Aye, K.M., Mon, N.C., Tan, T.C., Kumar, T., Onichandran, S. \& bin Mat Adenan (2014). Comparative study on Toxoplasma infection between Malaysian and Myanmar pregnant women. Parasites \& Vectors 7(564): 1-8. 
Brandon-Mong, G.J., Nurul Asma Anati, C.M.S., Sharma, R., Andiappan, H., Tan, C., Lim, Y.A.L. \& Nissapatorn, V. (2015). Seroepidemiology of toxoplasmosis among people having close contact with animals. Frontiers in Immunology 6(MAR): 1-6.

Castillo-Morales, V.J., Acosta Viana, K.Y., Guzmán-Marín, E.D.S., Jiménez-Coello, M., Segura-Correa, J.C., AguilarCaballero, A.J. \& Ortega-Pacheco, A. (2012). Prevalence and risk factors of Toxoplasma gondii infection in domestic cats from the tropics of mexico using serological and molecular tests. Interdisciplinary Perspectives on Infectious Diseases, 2012.

Cerro, L., Rubio, A., Pinedo, R., Mendes-deAlmeida, F., Brener, B. \& Labarthe, N. (2014). Seroprevalence of Toxoplasma gondii in cats (Felis catus, Linnaeus 1758) living in Lima, Peru. Brazilian Journal of Veterinary Parasitology 23(1): 90-93.

Chandrawathani, P., Nurulaini, R., Zanin, C.M., Premaalatha, B., Adnan, M., Jamnah, O., Khor, S.K., Khadijah, S., Lai, S.Z., Shaik, M.A., Seah, T.C. \& Zatil, S.A. (2008). Seroprevalence of Toxoplasma gondii antibodies in pigs, goats, cattle, dogs and cats in peninsular Malaysia. Tropical Biomedicine 25(3): 257-258.

Dabritz, H.A. \& Conrad, P.A. (2010). Cats and toxoplasma: Implications for public health. Zoonoses and Public Health 57(1): 34-52.

Deksne, G., Petrus, A. \& Kirjušina, M. (2013). Seroprevalence and Factors Associated with Toxoplasma gondii Infection in Domestic Cats from Urban Areas in Latvia. Journal of Parasitology 99(1): 48-50.

Dubey, J.P., Darrington, C., Tiao, N., Ferreira, L.R., Choudhary, S., Molla, B., Saville, W.J., Tilahun, G., Kwok, O.C. \& Gebreyes, W.A. (2013). Isolation of Viable Toxoplasma gondii from Tissues and Feces of Cats from Addis Ababa, Ethiopia. Journal of Parasitology 99(1): 56-58.
Dubey, J.P., Lindsay, D.S. \& Lappin, M.R. (2009). Toxoplasmosis and Other Intestinal Coccidial Infections in Cats and Dogs. Veterinary Clinics of North America - Small Animal Practice 39(6): 1009-1034.

Elmore, S.A., Jones, J.L., Conrad, P.A., Patton, S., Lindsay, D.S. \& Dubey, J.P. (2010). Toxoplasma gondii: epidemiology, feline clinical aspects, and prevention. Trends in Parasitology 26(4): 190-196.

Garcia, C.B.T., Jill, M., Talavera, P. \& Penul, G.M. (2014). Seroprevalence and Risk Factors Associated with Seropositivity to Toxoplasma gondii among Stray and Domestic Cats (Felis silvestris catus) in Metro Manila 26: 49-60.

Gauss, C.B.L., Almería, S., Ortuño, A., Garcia, F. \& Dubey, J.P. (2003). Seroprevalence of Toxoplasma gondii Antibodies in Domestic Cats from Barcelona, Spain Isolation and Genotyping of Toxoplasma gondii From Free-Ranging Chickens From Argentina. Jourmal of Parasitology 89(5): 1063-1064.

Györke, A., Opsteegh, M., Mircean, V., Iovu, A. \& Cozma, V. (2011). Toxoplasma gondii in Romanian household cats: Evaluation of serological tests, epidemiology and risk factors 102(4): 321-328.

Hill, D. \& Dubey, J.P. (2002). Toxoplasma gondii: Transmission, diagnosis, and prevention. Clinical Microbiology and Infection 8(10): 634-640.

Hooshyar, H., Rostamkhani, P., Talari, S. \& Arbabi, M. (2007). Toxoplasma gondii Infection in Stray Cats. Iranian Journal of Parasitology 2(1): 18-22.

Jittapalapong, S., Nimsupan, B., Pinyopanuwat, N., Chimnoi, W., Kabeya, H. \& Maruyama, S. (2007). Seroprevalence of Toxoplasma gondii antibodies in stray cats and dogs in the Bangkok metropolitan area, Thailand. Veterinary Parasitology 145(1-2): 138-141. 
Jones, C.D., Okhravi, N., Adamson, P. \& Tasker, S. (2000). Comparison of PCR Detection Methods for B1, P30, and 18S rDNA Genes of T. gondii in Aqueous Humor. Investigative Ophthalmology and Visual Science 41(3): 634-644.

Jung, B.K., Lee, S.E., Lim, H., Cho, J., Kim, D.G., Song, H., Kim, M.J., Shin, E.H. \& Chai, J.Y. (2015). Toxoplasma gondii B1 gene detection in feces of stray cats around Seoul, Korea and genotype analysis of two laboratory-passaged isolates. Korean Journal of Parasitology 53(3): 259-263.

Lappin, M.R. (2015). Feline intestinal protozoa - zoonotic importance or not? European Journal of Companion Animal Practice 25(1): 4-7.

Lee, S.E., Kim, N.H., Chae, H.S., Cho, S.H., Nam, H.W., Lee, W.J., Kim, S.H. \& Lee, J.H. (2011). Prevalence of Toxoplasma gondii infection in feral cats in Seoul, Korea. The Journal of Parasitology 97(1): 153-155.

Lilly, E.L. \& Wortham, C.D. (2013). High prevalence of Toxoplasma gondii oocyst shedding in stray and pet cats (Felis catus) in Virginia, United States. Parasites \& Vectors 6(1): 266.

Liu, Q.X., Wang, S., Wang, L.Q., Xing, J., Gao, W.J., Liu, G.F., Zhao, B., Zhang, H.B. \& Gao, L.H. (2014). Seroprevalence of Toxoplasma gondii infection in dogs and cats in Zhenjiang City, Eastern China. Asian Pacific Journal of Tropical Biomedicine 4(9): 725-728.

Mancianti, F., Nardoni, S., Ariti, G., Parlanti, D., Giuliani, G. \& Papini, R.A. (2010). Cross-sectional survey of Toxoplasma gondii infection in colony cats from urban Florence (Italy). Jourmal of Feline Medicine and Surgery 12(4): 351-354.

Millán, J., Cabezón, O., Pabón, M., Dubey, J.P. \& Almería, S. (2009). Seroprevalence of Toxoplasma gondii and Neospora caninum in feral cats (Felis silvestris catus) in Majorca, Balearic Islands, Spain. Veterinary Parasitology 165(3-4): 323-326.
Must, K., Hytonen, M.K., Orro, T., Lohi, H. \& Jokelainen, P. (2017). Toxoplasma gondii seroprevalence varies by cat breed. PLOS ONE 12(9): 1-10.

Nissapatorn, V. (2007). Toxoplasmosis: A Silent Threat in Southeast Asia. Research Journal Parasitology 2(1): 1-12.

Nissapatorn, V., Noor Azmi, M., Cho, S.M., Fong, M.Y., Init, I., Rohela, M., Khairul, A.A., Quek, K.F. \& Latt, H.M. (2003). Toxoplasmosis: prevalence and risk factors. Journal of Obstetrics and Gynaecology 23(6): 618-624.

Nutter, F.B., Dubey, J.P., Levine, J.F., Breitschwerdt, E.B., Ford, R.B. \& Stoskopf, M.K. (2004). Seroprevalences of antibodies against Bartonella henselae and Toxoplasma gondii and fecal shedding of Cryptosporidium spp. Giardia spp. and Toxocara cati in feral and pet domestic cats. Journal of the American Veterinary Medical Association 225(9): 1394-1398.

Oi, M., Yoshikawa, S., Maruyama, S. \& Nogami, S. (2015). Comparison of Toxoplasma gondii seroprevalence in shelter cats and dogs during 1999-2001 and 20092011 in Tokyo, Japan. PLoS ONE 10(8): 1-7.

Opsteegh, M., Haveman, R., Swart, A.N., Mensink-Beerepoot, M.E., Hofhuis, A., Langelaar, M.F. \& van der Giessen, J.W. (2012). Seroprevalence and risk factors for Toxoplasma gondii infection in domestic cats in The Netherlands. Preventive Veterinary Medicine 104(34): 317-326.

Paştiu, A.I., Györke, A., Villena, I., Balea, A., Páll, E., Spînu, M. \& Cozma, V. (2015). Comparative Assessment of Toxoplasma gondii Infection Prevalence in Romania Using 3 Serological Methods. Bulletin of University of Agricultural Sciences and Veterinary Medicine 72(1): 2013-2016.

Rengifo-herrera, C., Pile, E., García, A., Pérez, A., Pérez, D., Nguyen, F.K., Guardiam, V.D.L., Mcleod, R. \& Caballero, Z. (2017). Seroprevalence of Toxoplasma gondii in domestic pets from metropolitan regions of Panama. PARASiTE 24(9): 1-6. 
Robert-Gangneux, F. \& Dardé, M.L. (2012). Epidemiology of and diagnostic strategies for toxoplasmosis. Clinical Microbiology Reviews 25(2): 264-296.

Roqueplo, C., Halos, L., Cabre, O. \& Davoust, B. (2011). Toxoplasma gondii in wild and domestic animals from New Caledonia. Parasite 18: 345-348.

Saki, J. \& Khademvatan, S. (2014). Detection of Toxoplasma gondii by PCR and mouse bioassay in rodents of Ahvaz District, Southwestern Iran. BioMed Research International, 2014.

Salant, H., Markovics, A., Spira, D.T. \& Hamburger, J. (2007). The development of a molecular approach for coprodiagnosis of Toxoplasma gondii. Veterinary Parasitology 146(3-4): 214220.

Salant, H., Spira, D.T. \& Hamburger, J. (2010). A comparative analysis of coprologic diagnostic methods for detection of Toxoplama gondii in cats. American Journal of Tropical Medicine and Hygiene 82(5): 865-870.
Vollaire, M.R., Radecki, S.V. \& Lappin, M.R. (2005). Seroprevalence of Toxoplasma gondii antibodies in clinically ill cats in the United States. American Journal of Veterinary Research 66(5): 874-877.

Wang, S., Zhou, Y., Niu, J., Xie, Q., Xiao, T., Chen, Y., Li, H., Ma, C., Zhang, H., Liu, S. \& Zhang, Z. (2017). Seroprevalence of Toxoplasma gondii infection in domestic cats in central China. PARASiTE 24(10): 1-4.

Zewdu, E., Tessema, T.S., Tilahun, G., Cox, E. \& God, B. (2015). Comparison of agglutination test, microscopy and $\mathrm{nPCR}$ for diagnosis of Toxoplasma gondii isolated from sheep and goat of Central Ethiopia 19(1): 117-129. 